\title{
Atomic Resolution Characterization of Ni base Nanoparticles for Energy Devices.
}

\author{
H. A. Calderon ${ }^{1}$, F. Godinez-Salomon ${ }^{2}$, O. Solorza-Feria ${ }^{2}$, P. Specht $^{3}$, C. Kisielowski ${ }^{4}$ \\ ${ }^{1}$ Dept. Física, ESFM-IPN, Zacatenco D.F. 07338, Mexico. \\ ${ }^{2}$ Dept. Química, CINVESTAV, Mexico D.F., Mexico. \\ 3 JCAP and NCEM, LBNL, Berkeley, CA 94720, U.S.A. \\ ${ }^{4}$ Dept. Mats. Sci. Eng., UCB, Berkeley, CA 94720.
}

$\mathrm{Ni}$ base nanoparticles (NPs) are characterized under low dose conditions in TEM mode. These nanoparticles are mainly designed to act as catalysts in fuel cell devices but they are currently under evaluation for applications to use solar energy and produce a fuel (artificial photosynthetic devices, AP). Basically cost effective devices require the use of abundant materials and $\mathrm{Ni}$ base nanoparticles meet such a requirement. Here the investigation deals with $\mathrm{Ni}, \mathrm{NiO}$ and $\mathrm{Pt} @ \mathrm{NiO}$ nanoparticles that can be used either in fuel cells or FA devices. Particularly the use of Pt@NiO for solar cells is attractive for one of the important components of the FA device, namely the hydrogen evolution center, while NiO has been tested as a catalyst for the oxygen evolution center. Consequently an atomic characterization of the involved nanocrystals is of particular importance in order to understand the catalysis mechanism as well as to relate the catalyst structure to the corresponding activity. In this work, transmission electron microscopy is used with the objective to determine nature, shape and atomic distribution of $\mathrm{Pt}$ for different loadings $(0-30 \%)$ on a Ni core basis. In all cases the electron dose rate has been kept in the range 20-100 e- $/ \AA^{2} \mathrm{~s}$ in order to avoid surface rearrangement by interaction with the electron beam. The TEAM 05 has been used for this work. In all cases a focal series reconstruction procedure (EWR) is used to recover both phase and amplitude images that provide information of the spacing between atomic planes and the chemical nature of the corresponding atomic columns.

Two procedures have been used for synthesis of nanoparticles [1]. One of them produces Ni and the other NiO-NPs. NiO NPs are then covered with different loadings of Pt ranging from 8 to 16 at.\% in order to create incomplete core shell structures but with superior catalytic activity [1]. Figure 1 shows phase images of Ni NPs, their size varies from 1 to $7 \mathrm{~nm}$ and can agglomerate most likely due to their magnetic characteristics. The dose rate used to acquire the experimental images is $30 \mathrm{e}^{-} / \AA^{2}$ s. Figure 2 shows experimental images of NiO NPs acquired with a dose rate of $120 \mathrm{e}^{-} / \AA^{2} \mathrm{~s}$, their average size is around $1.5 \mathrm{~nm}$. During processing $\mathrm{Pt}$ is then deposited on $\mathrm{NiO}$ particles and a typical example is given in the phase images shown in Figs. 3a-d, the dose rate in these cases is around $55 \mathrm{e}^{-} / \AA^{2} \mathrm{~s}$ and the Pt coverage is nominally 8 at. \%. Figs. 3 a-d show mostly irregular nanoparticles shapes as made evident in Fig 3b. All nanoparticles can be seen with a negligible transformation due to the interaction with the electron beam. These NPs are nevertheless susceptible to alteration in shape and structure as a consequence of electron beam sample interaction [2]. An example is given in the phase images shown Figs. $3 \mathrm{~d}$-f. In these cases, the dose rate has been increased from $55 \mathrm{e}^{-} / \AA^{2} \mathrm{~s}$ (Fig. 3d) to $300 \mathrm{e}^{-} / \AA^{2} \mathrm{~S}$ (Fig 3e) and $1400 \mathrm{e}^{-} / \AA^{2} \mathrm{~s}$ (Fig. 3f). Clearly the particle under observation initially losses atoms that apparently redeposit on the carbon support and migrate (partially) to form a new crystal. The selected NP becomes bicrystalline at the end of this experiment that clearly shows the need to use a proper electron dosage for observation. Phase images have been used for simulation in order to determine the Pt coverage. 


\section{References:}

[1] F. Godinez Salomon et al., ongoing research.

[2] H. A. Calderon et al, publication in preparation.

[3] HACB and OSE whish to acknowledge support from CONACYT and IPN.
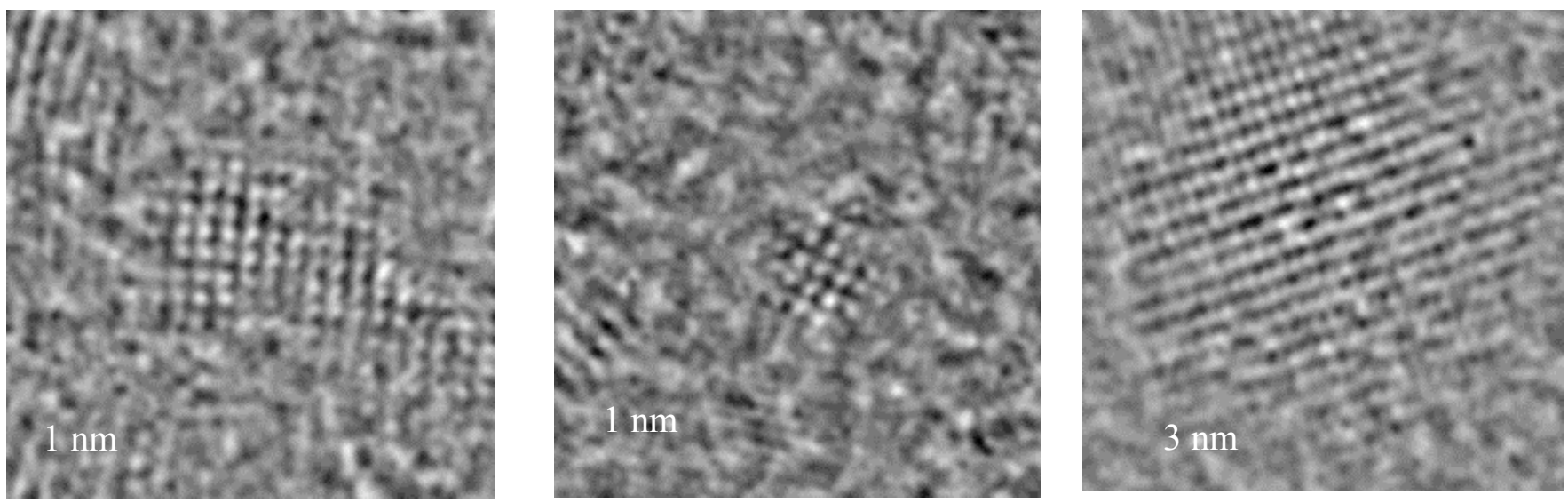

Fig. 1. Ni Nanoparticles, phase images after EWR procedure. A dose rate of $30 \mathrm{e}-/ \AA^{2} \mathrm{~s}$ is used.
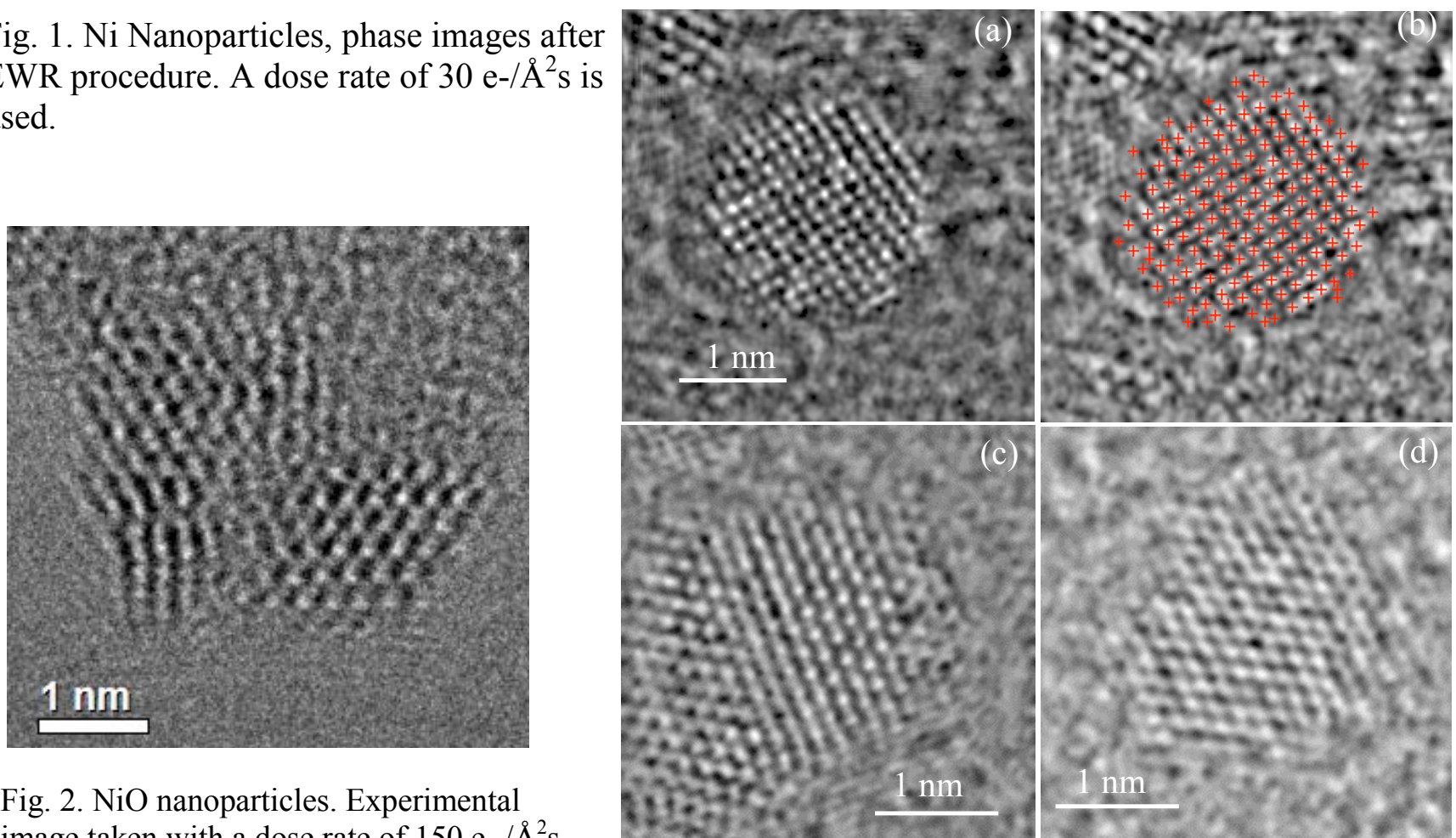

Fig. 2. NiO nanoparticles. Experimental image taken with a dose rate of $150 \mathrm{e}-/ \AA^{2}$ s.

Fig. 3. Phase images of $\mathrm{NiO}$ nanoparticles with a $\mathrm{Pt}$ coverage of approximately 8 at.\%. (a-d) Different nanoparticles taken with a dose rate of 55 e- $/ \AA^{2}$ s. Phase image of nanoparticle after $1 \mathrm{~min}$ at a dose rate of $300 \mathrm{e}-/ \AA^{2} \mathrm{~s}(\mathrm{e})$ and $1 \mathrm{~min}$ at a $1400 \mathrm{e}-/ \AA^{2}$ s. Images $(\mathrm{d}, \mathrm{e}, \mathrm{f})$ belong to same NP.
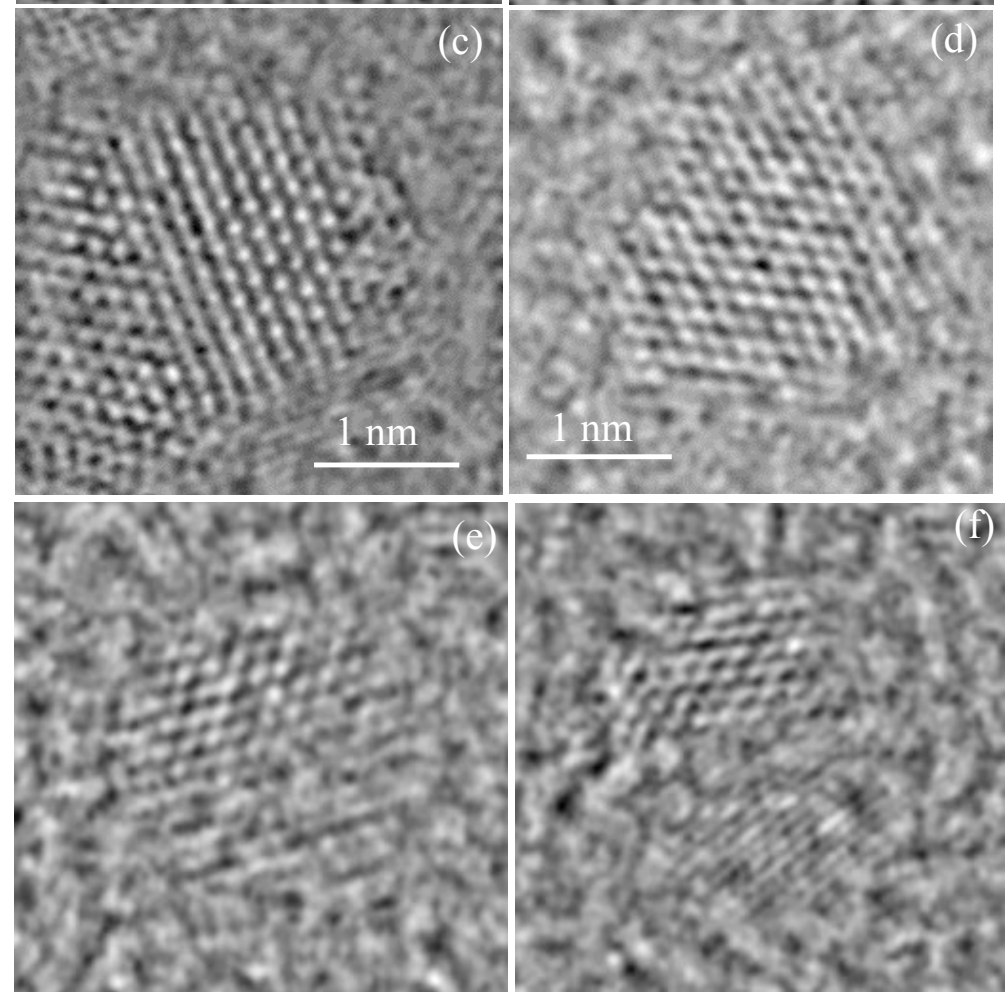